\title{
Combined Striatum, Brain Stem, and Optic Nerve Involvement due to Mycoplasma pneumoniae in an Ambulatory Child
}

\author{
Jin-Won Bae ${ }^{a}$ Hak-Jin Kim ${ }^{b}$ Gregory Youngnam Chang ${ }^{c}$ \\ Eun-Joo Kim ${ }^{\mathrm{a}}$ \\ Departments of a Neurology and ${ }^{b}$ Radiology, Pusan National University Hospital, \\ Pusan National University School of Medicine and Medical Research Institute, \\ Busan, Korea; 'Department of Neurology, University of California at Irvine Medical \\ Center, Orange, Calif., USA
}

\section{Key Words}

Mycoplasma pneumoniae $\cdot$ Basal ganglia $\cdot$ Optic neuritis

\begin{abstract}
In children, Mycoplasma pneumoniae encephalitis has been characterized by acute onset of an encephalopathy associated with extrapyramidal symptoms and symmetric basal ganglia with or without brain stem involvement on magnetic resonance imaging. Our case, showing unilateral optic neuritis, ophthalmoplegia, no extrapyramidal symptoms, and typical striatal involvement on magnetic resonance imaging, broadens the spectrum of varying clinical manifestations of childhood $M$. pneumoniae-associated encephalopathy.
\end{abstract}

\section{Introduction}

Cerebral complications of Mycoplasma pneumoniae often simulate other fulminant encephalitis in adults. Seizures and progressive obtundations are common [1]. Diagnosis is often overlooked since initial respiratory symptoms may be minimal or not present at all. In children, the diagnosis may be suspected when prominent symmetric striatum involvement with or without brain stem lesions is seen in the setting of an acute encephalitis $[2,3]$. Expected subcortical white matter lesions in acute disseminated encephalomyelitis may not be seen in M. pneumoniae encephalitis.

We recently encountered a child, who following M. pneumoniae, presented with left optic neuritis and abducens nerve palsy. Surprisingly, magnetic resonance imaging (MRI) 
showed extensive symmetric high-signal lesions, best seen on T2-weighted and fluidattenuated inversion recovery (FLAIR) images, involving striatum, midbrain, and pontine tegmentum. Right subcortical cerebellar white matter lesions and left optic nerve lesions were also seen. Discordant mild symptoms in presence of extensive MRI findings further broaden the spectrum of varying clinical manifestations of $M$. pneumoniae-induced cerebral dysfunction.

\section{Case Report}

A previously healthy 7-year-old boy was admitted complaining of blurry vision for 4 days. He had been diagnosed with $M$. pneumoniae 2 weeks before and had been recovering at home. His mother noted that over the course of 2 days, the boy took more naps than usual but remained playful despite visual complaints. She observed no difficulty with his ambulation. He denied any headache or eye pain. Neurological examination revealed an alert, responsive child with left abducens nerve palsy. His left visual acuity was limited to light perception. The right pupil was briskly reactive but the left was sluggish with afferent pupillary defect. Funduscopic examination confirmed a left swollen optic disc. He walked normally but complained of not seeing clearly. Laboratory examination was remarkable for elevated cold agglutinin of 1:32 and positive M. pneumoniae antibody titer of 1:2,560. Serum toxoplasma-specific IgM antibody, cytomegalovirus-specific IgM antibody, Rubella-specific IgM antibody, herpes simplex virus-specific IgM antibody and lactate levels were all negative or normal. Cerebrospinal fluid was normal with 0 white blood cells/dl, glucose of $56 \mathrm{mg} / \mathrm{dl}$, and protein of $37 \mathrm{mg} / \mathrm{dl}$. Oligoclonal banding was absent. MRI, on T2-weighted and FLAIR images, showed symmetric high-signal lesions over the putamen, pallidium, thalamus, and brain stem tegmentum near the floor of the 4th ventricle, but the subcortical white matter was spared (fig. 1a). A bright signal on the left optic nerve (fig. 1a, arrow) and a single right cerebellar white matter lesion were also detected. No abnormal signal change was seen on diffusion-weighted images. After starting intravenous methylprednisolone, his left visual acuity improved from light perception to finger count and abducens nerve palsy resolved over a 2-week period. Two weeks later, follow-up MRI showed improvement in attenuation of signal intensities (fig. $1 b)$.

\section{Discussion}

In adults, $M$. pneumoniae is known to have a wide range of clinical manifestations including encephalitis, myelitis, cranial neuropathy, optic neuritis and Guillain-Barré syndrome [4]. Cerebral involvement may range from transient meningitis to an acute fulminant encephalitis requiring life saving craniectomy [5]. However, in children, central nervous system involvement has been more stereotypically characterized by onset of an acute encephalopathy associated with an extrapyramidal syndrome, and MRI showing predominant symmetric basal ganglia [2] with or without brain stem involvement [3]. Our patient is unique in that he presented mainly with visual disturbance from left optic neuritis and abducens nerve palsy in absence of encephalopathy or motor dysfunction.

Pathologic examinations in encephalopathy induced by M. pneumoniae have disclosed focal evidence of necrosis, demyelination and immune-mediated vasculopathy [4]. Delayed onset of neurologic symptoms following $M$. pneumoniae, absence of spinal fluid pleocytosis, rapid recovery of optic neuritis following steroid therapy, and normal diffusion-weighted images suggest immune-mediated demyelination as the predominant mechanism in this child.

Extensive symmetric striatal involvement suggests metabolic or infectious processes. Saitoh et al. [2] have reported an 11-year-old boy with progressive encephalitis with 
ophthalmoplegia and extrapyramidal syndrome with symmetric striatal lesions on MRI. Serum antimycoplasma antibody titers were markedly elevated, and cerebrospinal fluid polymerase chain reaction analysis for M. pneumoniae was positive. The striking similarity of the MRI of Saitoh et al.'s case with our case suggests that concomitant immune-mediated demyelination and direct infection may have roles in some childhood M. pneumoniae cases.

Our case, presenting with optic neuritis and ophthalmoplegia, but with typical MRI findings of extensive symmetric striatal involvement, broadens the spectrum of various clinical manifestations of childhood M. pneumoniae-associated encephalopathy.

\section{Acknowledgements}

This study was supported by a grant of the Korea Health Technology R\&D Project, Ministry of Health and Welfare, Republic of Korea (A070001), and a clinical research grant from Pusan National University Hospital 2011. 

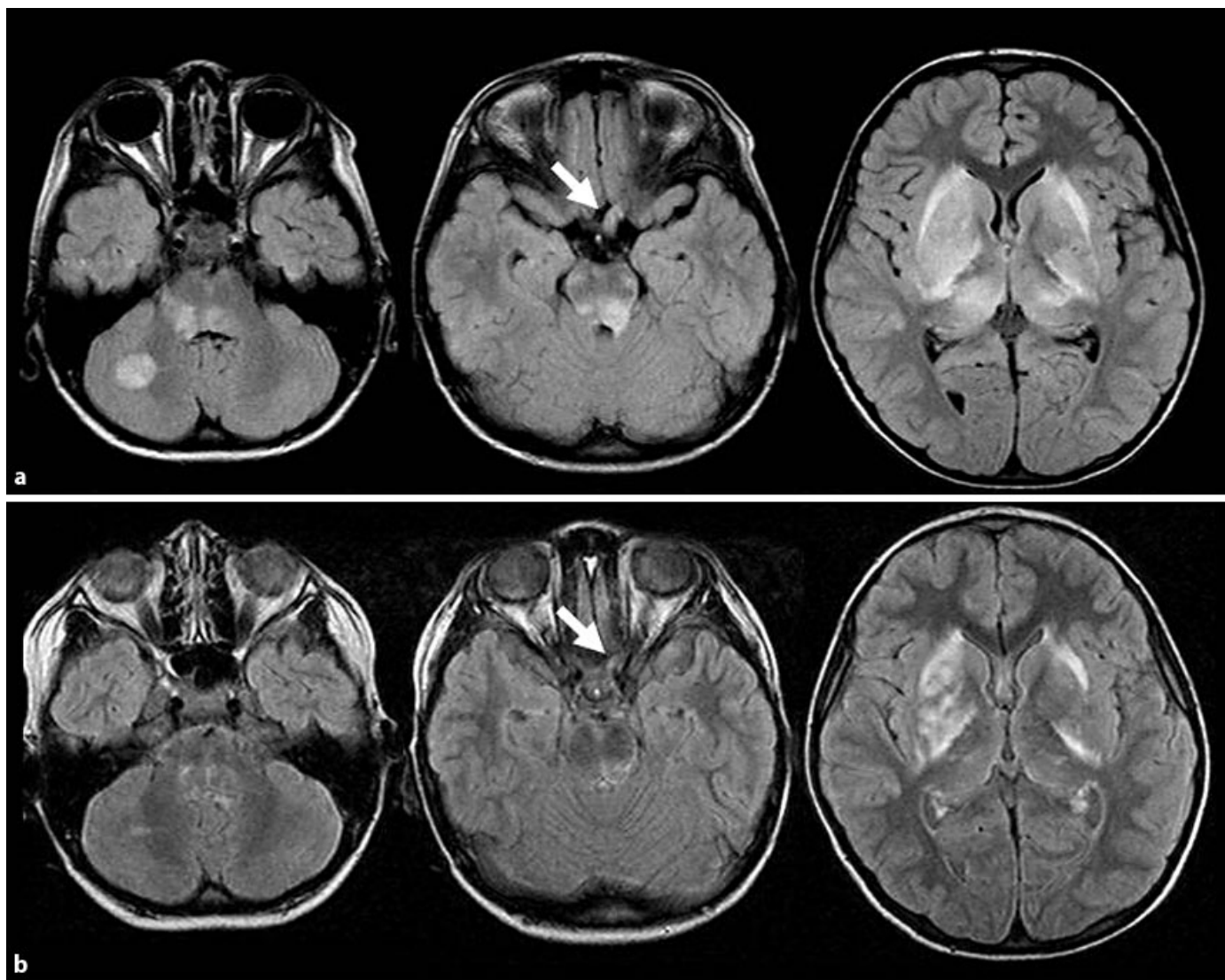

Fig. 1. a Initial axial FLAIR images demonstrate marked high-signal intensity in the right cerebellum, brain stem, left optic nerve (arrow), bilateral basal ganglia and thalamus. b Follow-up FLAIR images at 2 weeks after treatment show decreased high-signal intensities compared to those of the previous images.

\section{References}

1 Bitnun A, Ford-Jones EL, Petric M, MacGregor D, Heurter H, Nelson S, Johnson G, Richardson S: Acute childhood encephalitis and Mycoplasma pneumoniae. Clin Infect Dis 2001;32:1674-1684.

-2 Saitoh S, Wada T, Narita M, Narita M, Kohsaka S, Mizukami S, Togashi T, Kajii N: Mycoplasma pneumoniae infection may cause striatal lesions leading to acute neurologic dysfunction. Neurology 1993;43:2150-2151.

-3 Sakoulas G: Brainstem and striatal encephalitis complicating Mycoplasma pneumoniae pneumonia: possible benefit of intravenous immunoglobulin. Pediatr Infect Dis J 2001;20:543-545.

4 Narita M: Pathogenesis of neurologic manifestations of Mycoplasma pneumoniae infection. Pediatr Neurol 2009;41:159-166.

5 Schwab S, Jünger E, Spranger M, Dörfler A, Albert F, Steiner HH, Hacke W: Craniectomy: An aggressive treatment approach in severe encephalitis. Neurology 1997;48:412-417. 